\title{
The Complexity and Application of Syntactic Pattern Recognition Using Finite Inductive Strings
}

\author{
Elijah Myers ${ }^{1}$, Paul S. Fisher ${ }^{1}$, Keith Irwin $^{1}$, Jinsuk Baek ${ }^{1}$, and Joao Setubal ${ }^{2}$ \\ 1 Department of Computer Science, Winston-Salem State University, USA \\ \{emyers106, fisherp, irwinke, baekj\}@wssu.edu \\ 2 Viginia Bioinformatics Institute, Virgina Tech Blacksburg, VA 24061, USA \\ setubal@vbi.vt.edu
}

\begin{abstract}
We describe herein the results of implementing an algorithm for syntactic pattern recognition using the concept of Finite Inductive Sequences (FI). We discuss this idea, and then provide a big O estimate of the time to execute for the algorithms. We then provide some empirical data to support the analysis of the timing. This timing is critical if one wants to process millions of symbols from multiple sequences simultaneously. Lastly, we provide an example of the two FI algorithms applied to actual data taken from a gene and then describe some results as well as the associated data derived from this example.
\end{abstract}

Keywords: Pattern Recognition, finite induction, syntactic pattern recognition, algorithm complexity.

\section{Introduction}

Despite the fact that there has been extensive research and development within the pattern recognition topic, new problems continue to emerge that require more efficient revisions of existing techniques and, occasionally, new techniques to solve existent problems. For example, the problems associated with finding motifs [1, 2. are particularly difficult due to mutations, unknown boundaries, etc. While many new problems continue to emerge that could potentially benefit from the use of pattern recognition, but the current effort reported herein is an extension with applications of previous work [3] in reference to the field of bioinformatics, where it is often the case that genetic data is processed for a vast multitude of diverse purposes. Regardless of the purpose of the research, bioinformatics often entails processing genetic data in the form of strings consisting of the symbols $A, C, G$, and $T$ as well as equivalent protein sequences. This type of string is suitable for syntactic pattern recognition using finite inductive (FI) sequences, but again there are some issues that need to be addressed, and we will address some of them later in this paper. It is the purpose of the FI algorithms [4] to provide a general technique to achieve pattern recognition when comparing finite strings in order to determine a) what patterns exist in the examined strings, and b) whether or not subsequent strings contain similar or identical subsequences in the same form as such exemplar substrings are known by the algorithms.

T.M.H. Dijkstra et al. (Eds.): PRIB 2010, LNBI 6282, pp. 125-136, 2010.
(C) Springer-Verlag Berlin Heidelberg 2010 


\section{Review of FI Algorithms and Theory}

The idea 4 is to introduce a 'ruling' as a finite machine that can, when provided a short driving sequence, generate a sequence that is much longer. The 'rules' called implicants contained within the ruling come from the processing of a finite sequence of symbols constructed from the designated alphabet. We further stipulate that the choice of any symbol at any particular position depends upon only the symbols at the previous $n$ points. The least such $n$ is called the inductive base (IB) for the sequence. We define an implicant as the pair $(w, p)$ consisting of a word $w$ over the alphabet and a single member $p$ of that alphabet. We also require that $\mathrm{w}$ occurs at least once, and whenever $w$ occurs, then it is followed immediately by $p$. We express this relationship as $w \rightarrow p$, and call $w$ the antecedent and $p$ the consequent. We also assume $w$ is in reduced form: there is no proper terminal segment that is the antecedent of another implicant. We can state the following simple properties:

- For any finite sequence, the IB is the maximum length of the antecedents in the reduced form implicants.

- If an FI sequence has inductive base $A$ and contains $b$ symbols in the alphabet, then the upper bound for the reduced form implicants is $b^{A}$.

For purposes of simplicity, we will assume there is a distinguished symbol $S$ that serves as the start symbol for all FI sequences. We also state without proof that if the original implicants (called prime implicants) generated from the sequence have inductive bases that differ among themselves, then it is possible to reduce the inductive base $b$ of the implicants to a value $1 \leq \mathrm{IB}<b$.

\subsection{Generating and Applying the FI Algorithms}

There are two algorithms that make up the FI system. These are called Factoring and Following. Factoring is the process whereby a storage structure called Ruling is generated based upon an a'priori IB, and Following is the process whereby the ruling is applied to unknown patterns.

Example 1: Factoring. Suppose we have a sequence aactgctagt. We append the start symbol and then begin the process of factoring, and we will allow the IB to be as large as necessary to accommodate all of the implicants in one level (called Prime Implicants).

Input Sequence: Saactactagt

Implicants: $S \rightarrow$ a, $S$ a $\rightarrow$ a, aa $\rightarrow$ c, ac $\rightarrow$ t, ct $\rightarrow$ a, aacta $\rightarrow$ c, tac $\rightarrow$ t, tacta $\rightarrow \mathrm{g}$, and $\mathrm{g} \rightarrow \mathrm{t}$

As can be seen from the implicants, the IB is 5 , and there are other implicants with IB less than 5 , so we can reduce these prime implicants to new implicants with IB say 2. We do so in the following steps:

Step 1: We note that the following implicants meet our new IB value of 2:

$$
S \rightarrow \mathrm{a}, S \mathrm{a} \rightarrow \mathrm{a}, \mathrm{aa} \rightarrow \mathrm{c}, \mathrm{ct} \rightarrow \mathrm{a}, \mathrm{ac} \rightarrow \mathrm{t}, \mathrm{g} \rightarrow \mathrm{t}
$$


This leaves the symbols in the string from (1) as follows in (3) where the consequents not kept (pushed out) are shown in Level 1:

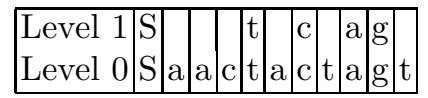

Step 2: We apply the same process of Step 1 to the symbols remaining in Level 1. Level 1 is called the residual for Level 0 . This produces the following rules (4) with an empty residual:

$$
S \rightarrow \mathrm{t}, \mathrm{t} \rightarrow \mathrm{c}, \mathrm{c} \rightarrow \mathrm{a}, \mathrm{a} \rightarrow \mathrm{g}
$$

From (2) and (4) we can now define the ruling with inductive base 2 for the sequence aactactagt with driving sequence $S$.

$$
\begin{array}{|l|l|c|c|c|c|c|}
\hline \text { Level 1 } & S \rightarrow \mathrm{t} & \mathrm{t} \rightarrow \mathrm{c} & \mathrm{c} \rightarrow \mathrm{a} & \mathrm{a} \rightarrow \mathrm{g} & & \\
\text { Level 0 } & S \rightarrow \mathrm{a} & S \mathrm{a} \rightarrow \mathrm{a} & \mathrm{aa} \rightarrow \mathrm{c} & \mathrm{ct} \rightarrow \mathrm{a} & \mathrm{ac} \rightarrow \mathrm{t} & \mathrm{g} \rightarrow \mathrm{t} \\
\hline
\end{array}
$$

Example 1: Following. Suppose we have a new sequence $S$ aactggacattac and we want to process it against our known sequence as represented by the ruling in Example 1.

Step 1: We apply all implicants of (5) in Level 0 to see for the given antecedents if the consequent matches. If it matches, then the consequent is deleted at the end of the processing for this level. The symbols bolded indicate that the symbol is deleted.

$S$ a a c t g g a c a t t a c

Step 2: We apply the implicants of (5) in Level 1 to the residual of Step 1.

$$
S \text { g g a c a t t a c }
$$

This results in the residual string $S \mathrm{~g} g$ a c t t a c. One cannot say much about the two strings as how they relate to one another, since they do not represent much data; but we can say in general that the two sequences are not very similar to one another. At this stage, we could add the sequence of Example 2 to the ruling, if it was important. In general, the Factoring process can deal with $n$ sequences simultaneously, so we can deal with permutations of sequences if they are important. We can also make the rules non-deterministic.

\section{Algorithm Overview}

Before carrying out an empirical analysis of the implemented FI algorithms, we first consider how the algorithms were implemented so the analysis will be understood. Implementation decisions for the general version of the algorithm were 
based on the following two principles: (1) the performance of the algorithm will be greatly enhanced if we can ensure linear runtime, and (2) the algorithm must be implemented so it can be applied to strings of considerable length without causing problems related to memory consumption.

\subsection{Factoring Analysis}

The first step in the factoring portion of the algorithm is to read each of the valid symbols in the alphabet from a file, store them in an array that holds all the alphabet symbols, and assign each one a numerical value based upon the index where the symbol was stored. The total number of symbols in the alphabet is $b$, called the base, due to the fact that alphabet sequences are treated as a numbers represented in the corresponding base number system for the purposes of hashing. In order to implement hashing, a second array of size $b^{I B}$ is created (IB again is the maximum antecedent length defined as inductive base) with each index representing a possible rule antecedent. All indices are initialized to a null value that indicates that the antecedent does not yet have a corresponding consequent. Symbols from the initial string are read one at a time and placed in a queue that maintains the previous symbols read, up to the maximum value of inductive base.

Once the queue fills for the first time each new symbol that is read is treated as the consequent of the antecedent that is implied by the contents of the queue. The sequence of symbols that currently fill the queue are hashed to determine the index of the corresponding antecedent. The current symbol is then compared to the contents of that index; if the index is empty the consequent is placed at the corresponding index. If the index already contains a matching consequent no action is taken. If the index contains a consequent that does not match, the current symbol in the index is given a special value that indicates that the antecedent represented by that index is not a valid antecedent. The indices that are generated by each hash are written to a temporary file each time a hash occurs to serve as input for the next step of the process.

With every possible antecedent having been marked as empty, invalid, or containing a valid consequent, the process of generating the residual for the next level of processing can begin. Each hash index that was previously written to the temporary file is read while simultaneously examining the symbol from the initial string that was being examined when the hash occurred. If the hashed address points to an antecedent index that has been flagged as invalid, the symbol is written to a file as part of the string that will be factored in the next level. With the new string generated for the next level, the remaining task is to output the valid rules based upon the antecedents that have valid consequents in their reduced form. In order to reduce each rule before outputting it, the task of examining every antecedent that could keep the rule from being reduced must be performed (i.e. the rule $B A \rightarrow B$ can be reduced to $A \rightarrow B$ as long as $A A$ $\rightarrow B, B A \rightarrow B, C A \rightarrow B$, and $D A \rightarrow B$ are all true or do not exist if the input alphabet consists only of $A, B, C$ and $D$ ). It is sufficient to state that this can be accomplished by examining the contents of the array that symbolizes all 
antecedents once for each reduction that is to take place (i.e. a maximum IB of length three would require two passes through this array to find any rules that can be reduced to IB of length one). The entire process described to this point must be repeated for each ruling level that is generated with the exception of loading the alphabet, which occurs once.

\subsection{Following Analysis}

The following process is far simpler in design than factoring. The alphabet must still be initially loaded, and the array to represent all possible antecedents must still be initialized so that each antecedent is empty. The first ruling level is read from a file and each valid rule that was found is processed so that the consequent is placed in the appropriate index that corresponds to its antecedent. Once the array of antecedents has been filled, the process of reading the target string one symbol at a time, similar to reading the input string in factoring, is performed, in the same manner as the factoring process. The only difference is that an empty antecedent or one that contains a consequent that does not match the current symbol will result in the current symbol being written to a file as a residual for this level. Each new corresponding level requires the repetition of this process with the ruling appropriate for that level and the new target string that was generated by processing the previous target string using the previous level's ruling. Thus when the process is complete the user is left with a file that contains all of the symbols that did not conform to any implicants in the previous level of the ruling.

\section{Performance Analysis}

With a general understanding of how the factoring and following processes are implemented, we consider the anticipated performance of the algorithm. We are interested in determining if the algorithm as described can be processed in linear time. The size of the input is the primary consideration, and expected runtimes are expressed in terms of input volume.

The factoring process is the more complicated and will necessarily have the longest performance time. The initial pass through the alphabet can be represented in terms of $b$, the number of valid symbols in the alphabet (also known as the base). The size of the array to represent all possible rule antecedents, namely $b^{I B}$, must be counted each time the array is examined. This occurs once when initializing each antecedent as empty, and once for each pass to determine if rules can be reduced (IB - 1). Thus we can represent this element of performance by the formulation (IB - 1) $\left(b^{I B}\right)+b^{I B}$. The length of the initial string we will designate by $N$. The entire length is processed three times for each level: once while scanning symbols to generate consequents, once while examining the hash addresses that were output, and a third time while examining the hash addresses. This requires $2 N$ time. Taken together, the algorithm's performance for processing a single level can be described by $b+b^{I B}+(\mathrm{IB}-1)\left(b^{I B}\right)+$ 
$3 N$. We know this process is repeated with each ruling level $L$ that is generated, except when loading the alphabet. This estimate is shown in (6).

$$
b+L\left[b^{I B}+(I B-1)\left(b^{I B}\right)+3 N\right]
$$

For following we need to determine the elements that are factors in its expected performance. The alphabet must still be loaded, and this can again be expressed by the variable $b$. The antecedent array still exists and can still be represented by $b^{I B}$; however, the number of times that this array is examined differs. The array is still initialized once while processing the current level, but now it is only examined one additional time as rules are expanded back into their maximum antecedent form. This can be represented simply as $2 b^{I B}$. The length of the compared string, $N$, is only examined once per level in the process of following, but we must define a new variable $R$ that corresponds to the number of rules in the ruling for the level, since each rule must be loaded into the antecedent array. This yields the upper bound on time complexity of $b+R+2 b^{I B}+N$ for each level and (7) for all levels.

$$
b+L\left[R+2 b^{I B}+N\right]
$$

Expressing these equations in terms of Big-O notation yields the equations (8) and (9) for the factoring and following respectively.

$$
\begin{gathered}
\mathrm{O}\left(b+L\left[b^{I B}+(I B-1)\left(b^{I B}\right)+3 N\right]\right) \\
\mathrm{O}\left(b+L\left[R+2 b^{I B}+N\right]\right)
\end{gathered}
$$

We can substitute $b^{I B}$ in for $R$ since this value is the maximum value. Next expanding (8) yields (10), and with the substitution, (9) can be rewritten as (11).

$$
\begin{gathered}
\left.\mathrm{O} b+L\left[I B b^{I B}+3 N\right]\right) \\
\mathrm{O}\left(b+L\left[3 b^{I B}+N\right]\right)
\end{gathered}
$$

While the term $b$ is variable depending on the problem domain, the value remains constant within any single problem domain (i.e. the algorithm is not designed to apply rulings to strings that are formed from a different alphabet than the string that was examined during the factoring process). Furthermore, the algorithm always utilizes the same maximum IB in the following process as the one used in the factoring process (i.e. the algorithm may be applied to strings of any length, but the maximum IB does not vary between the factoring and following processes). It is therefore possible, based on these facts and the fact the variables are independent of $N$, to treat these variables as constants. Removing these variables, along with all other constants from both equations, produces the equation (12) for both factoring and following.

$$
\mathrm{O}(L+L N)
$$


The worst-case scenario of the FI Algorithm can be determined by examining cases where the process of factoring is applied to a string that contains few recognizable patterns or no patterns at all. In the latter case it is evident that the only rule that can possibly be generated is the rule that defines those symbols that start the given string. When this type of string is factored it will create a situation where the number of levels in the ruling will be $\frac{N}{b}$ bounded by $N$ if $b$ is small. Knowing this fact leads to the conclusion that the worst-case scenario of the FI Algorithm is one where the factored string is entirely random, and the expected performance time $(\mathrm{O}(L+L N))$ is quadratic $\left(\mathrm{O}\left(N+N^{2}\right)\right)$. We now show a strategy to prevent this scenario from occurring.

The expected performance of (12) for the factoring and following portions of the algorithm can be reduced to $\mathrm{O}(N)$ under one of the two following conditions: 1) we can ensure that the term $L$ remains a constant, or 2) we can ensure that $L$ remains an insignificant factor when compared to the variable $N$. It is possible to begin to satisfy the second condition by restricting the strings being factored by the algorithm to only those that are believed to have significant underlying patterns. However, this is idealistic in the sense that the randomness of the factored string would have to be determined beforehand in order to ensure that this fact remains true. It is a simpler task to allow the user to place an upper bound on the number of levels that can be produced by factoring the given string, thus ensuring that $L$ remains a constant that is equal to or less than this upper bound; it is this strategy that is employed to ensure a linear runtime of the proposed algorithm.

\section{Empirical Performance Test}

The following subsections discuss the empirical results of the experiment in order to determine the accuracy of the predicted expectations that both processes will perform with linear performance dependent upon the number of input symbols $N$.

\subsection{Experiment Design}

The experiment is designed to allow for the testing of whether or not the factoring and following are producing linear runtimes. In order to fulfill these testing requirements, the processes of factoring and following have been executed input data sizes increasing by an order of magnitude (i.e. string lengths of $10^{3}$, $10^{4}, 10^{5}, 10^{6}, 10^{7}$, and $10^{8}$ symbols) using an alphabet that consists of four symbols: $A, B, C$, and $D$. In order to determine linear performance regardless of the maximum IB used two IBs (5 and 10). The maximum number of levels that can be processed has been restricted to 100 levels in all cases, and each process is implemented in $\mathrm{C}++$ with the timing mechanism built into the code itself. 


\section{$5.2 \quad$ Timing Results}

Ten repetitions were done for each experiment with times recorded to the nearest millisecond. Fig. 1 and 2 provide summary results of these experiments for factoring and following respectively. The first task in analyzing the resultant data from the experiment is to determine if the factoring portion of the algorithm is indeed producing slower execution times than the following portion of the algorithm. The graphical data suggests that the factoring process is producing slower times compared to the following process, but the question remains as to how much slower. In analyzing the raw data we obtained from the repeated experiments, we can compare the performance of the factoring portion to the following portion of the algorithm. Our empirical results show that the factoring process required 5.82 times the execution time of the following process when we are dealing with the longer antecedents and a value of 2.49 times for the shorter antecedents.

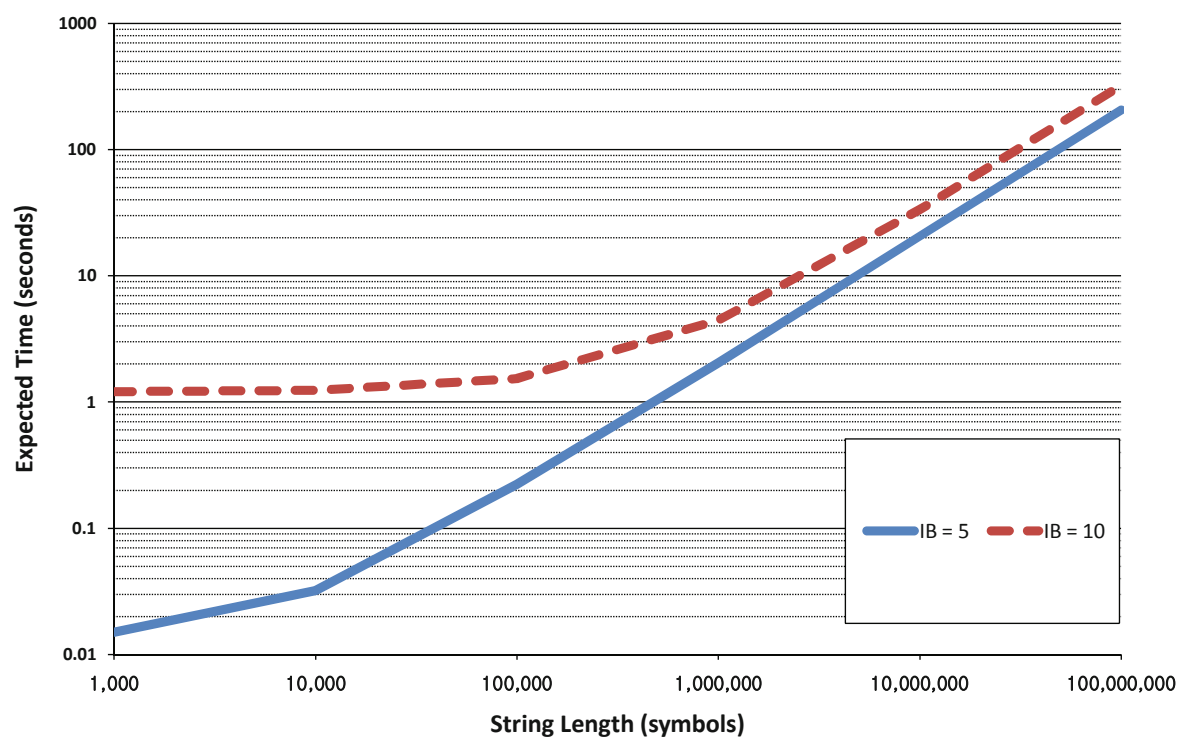

Fig. 1. Data for the Factoring process

The data recorded in both processes that correspond to a maximum IB of length five clearly produced a linear progression (as demonstrated by Fig. 1 and 2 ), despite the fact that there is an increase in the slope of the line that corresponds to the factoring process once the length of the target string exceeds $10^{4}$ symbols. A linear progression is also reached in both processes using a maximum IB of length ten, but the progression does not become completely linear until the length of the target string has reached $10^{6}$ and $10^{5}$ symbols for factoring and following, respectively. 


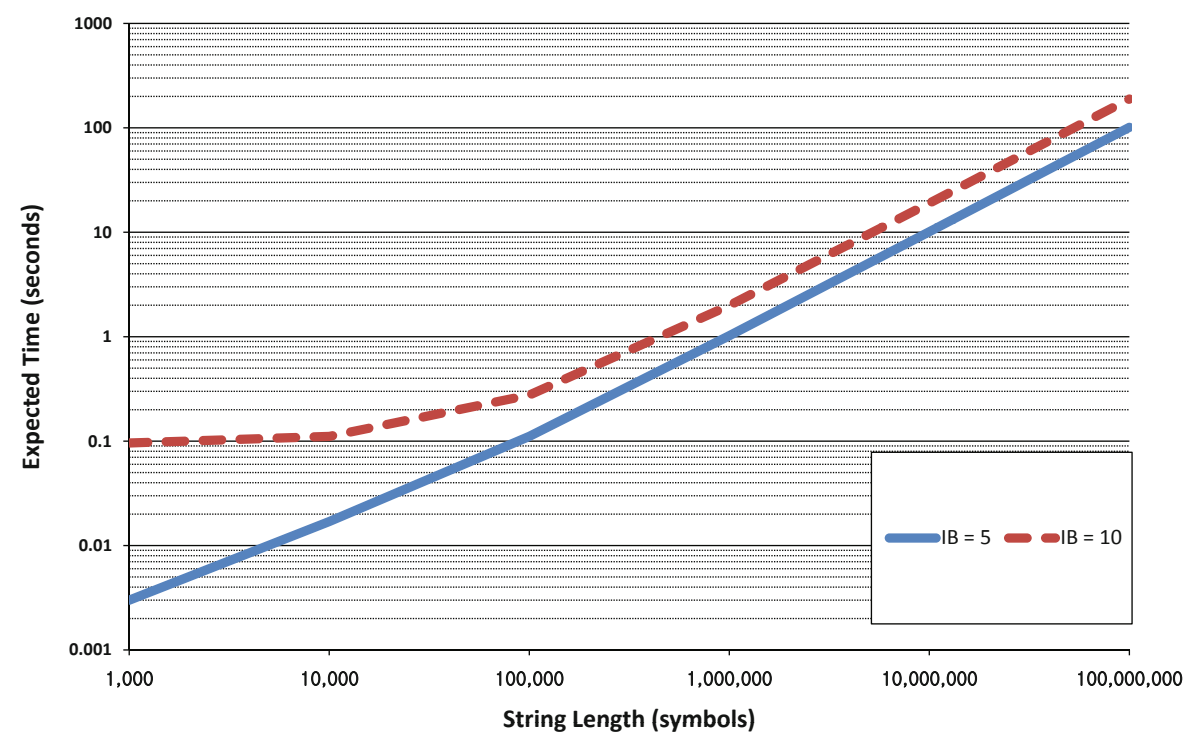

Fig. 2. Data for the Following process

\section{Application of FI Algorithm to Actual Data}

Consider the nucleotide subsequence obtained from [5] shown in (13).

\section{GTGCGATTTTTTTCTCCTCCTTTTTTTACCCTCCCGTT

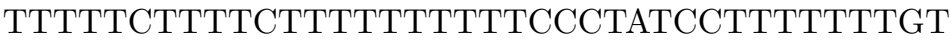

This subsequence begins at the $244^{\text {th }}$ position in the sequence consisting of some 21,069 symbols. Factoring this sequence we obtained 16,916 rules, meaning that the sequence has 4,153 nucleotides that have duplicate antecedents. This implies $20 \%$ of the subsequences overlap at least with one other subsequence. The ruling built consists of 18 levels, and since this percentage comes from a multilevel ruling, this commonality between subsequences may be due to elimination of symbols at one level producing homogeneous antecedents at the next level. Fig. 3 shows the number of implicants by level. From Fig. 3, we see that the number of implicants stays pretty constant through level 8, and then it grows quickly as the levels increase. This growth can be attributed to the fact that the sequence becomes choppier, that is, the repeated runs of patterns are removed by level 9 and so with more disparity, fewer identical antecedents with differing consequents contradict one another.

From Fig. 3, we see that the number of implicants stays pretty constant through level 8, and then it grows quickly as the levels increase. This growth can be attributed to the fact that the sequence becomes choppier, that is, the repeated runs of patterns are removed by level 9 and so with more disparity, fewer identical antecedents with differing consequents contradict one another. For the 


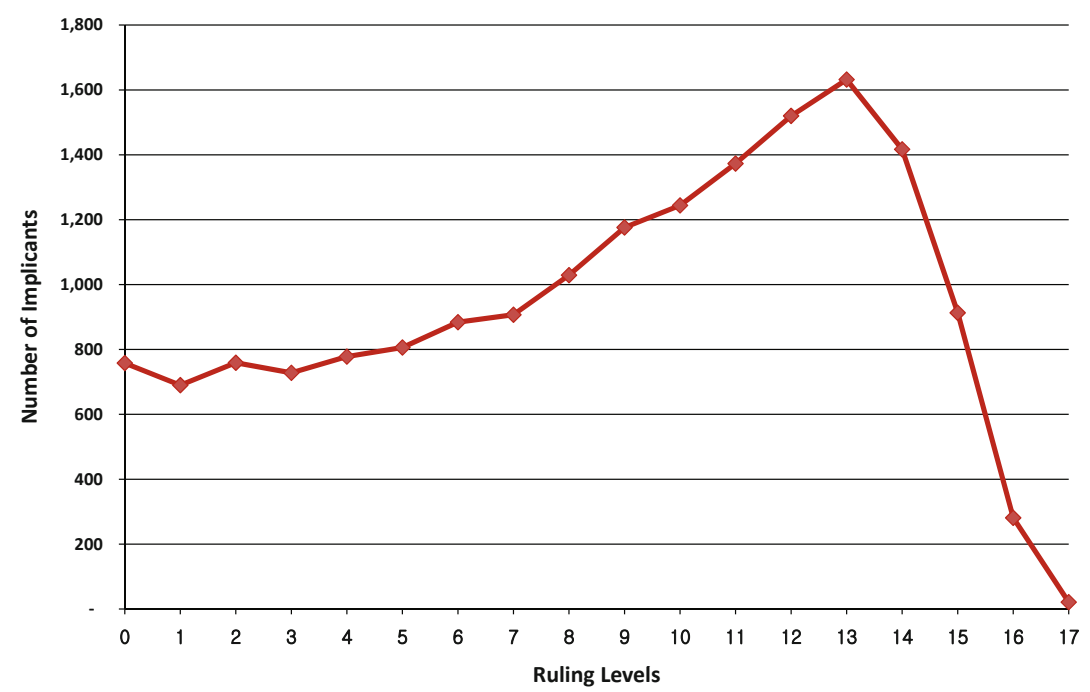

Fig. 3. Number of implicants per each level in the ruling

next step, we factored the subsequence in (13) and used this as the ruling with an inductive base of length 3 , and then processed the entire sequence. Fig. 4 provides the results of this activity. The light gray cells are those that belong to (13), and the dark gray are those symbols that have an implicant matching one in the ruling but are not in the subsequence of interest. The basis for this type of application is well treated in [6].

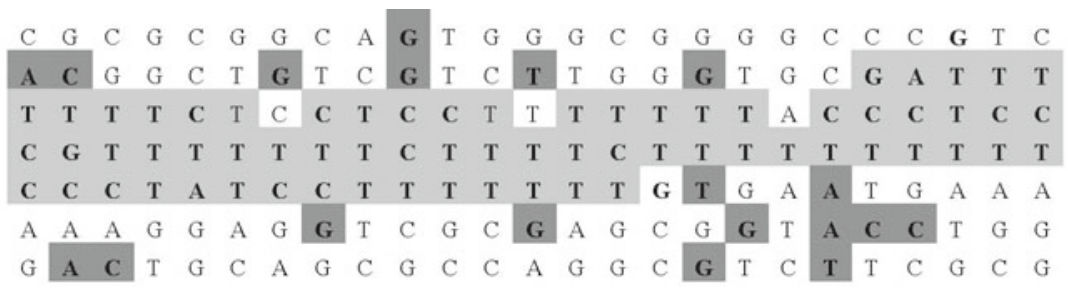

Fig. 4. Nucleotide sequence starting at position 201 and ending at position 375

Besides the subsequence in Fig. 4, there are other matches. Fig. 5 shows two of the longer ones. The first subsequence in Fig. 5 starts at position 54 and the second starts at position 935. Since the identification of a matching substring is not difficult, we provide an extension to the matching under random permutations of the subsequence being used to build the search ruling. We modified $10 \%$ of the symbols in the substring we were looking for and then followed the unknown string with a ruling of inductive base 3 and another with inductive base 9 . We 


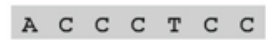

\section{$\begin{array}{llllllllllllll}\text { A } & \text { T } & \text { C } & \text { C } & \text { T } & \text { T }\end{array}$}

Fig. 5. Two subsequences found by the ruling with white indicating no match

provide the results in Fig. 6, where we only show that portion associated with the location of the substring we are trying to find. In Fig. 7 we show another contiguous substring from the unknown string, so that the density of the two areas can be compared. Comparing the results from these two test blocks, we obtain the results shown in Table 1 . We have also compared the complexity of such nucleotide sequences by considering their representation within a ruling as a measure not unlike Kolmogorov complexity [7].

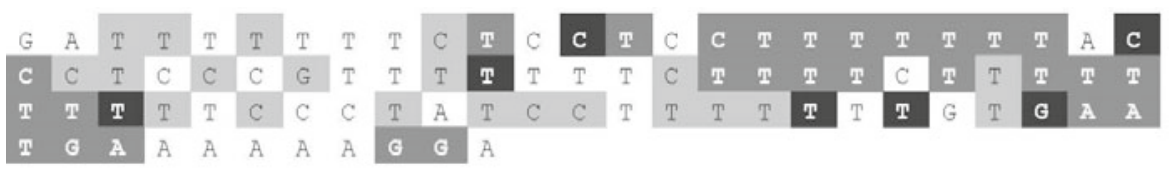

Fig. 6. Results of Following when $10 \%$ of the symbols are changed. White indicates no change, dark gray are symbols recognized by ruling of IB of 9 , light gray are recognized symbols by ruling of IB 3, and $50 \%$ gray are symbols recognized by both rulings.

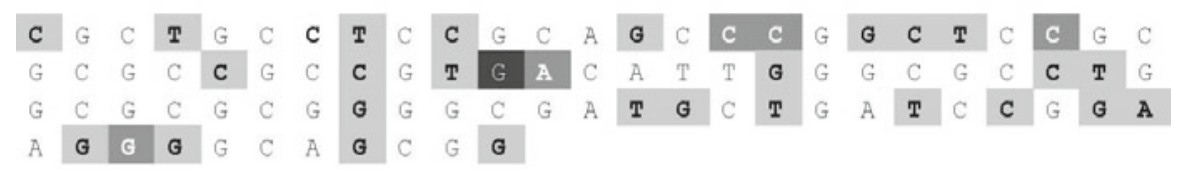

Fig. 7. Results starting from position 450 in the unknown sequence where the color key is identical to that of Fig. 6

Table 1. Comparative counts for the data of Fig. 6 and 7

\begin{tabular}{|l|c|c|c|c|}
\hline & IB & IB & & \\
\hline & 3 & 9 & Both & White \\
\hline Fig. 6 & 22 & 7 & 26 & 30 \\
Fig. 7 & 26 & 1 & 4 & 54 \\
\hline
\end{tabular}

\section{Conclusion}

The results of this work have been to provide a comparative basis for the timing of an algorithm that will recognize substrings of symbols, even under mutation. We have shown by logical argument as well as by empirical data that the algorithm operates in linear time with the size of the input data sequence being the 
driving factor. Also as shown in the last section in Table 1, the short inductive base provides too many extraneous symbol matches, while the long inductive base provides too few. There must be another inductive base that would be most appropriate. But even with these two selections, and the selections were made to first yield a ruling with only one level $(\mathrm{IB}=9)$ and as many levels as we could obtain $(\mathrm{IB}=3)$, the results provide an upper and lower bound. We still have more work to do to refine this algorithm to provide a more robust result for processing large sequences of symbols from a small alphabet. It is clear that the longer the sequence, the more potential there is for conflict when the inductive base is fixed to a reasonable value, perhaps 5 to 7 symbols. We have shown that such conflicts indeed do exist in the early levels of a ruling, limiting their growth. Lastly, we believe that the approach of non-deterministic rulings may provide an additional benefit for this kind of processing.

\section{References}

1. Buhler, J., Tompa, M.: Finding Motifs Using Random Projections. Journal of Computational Biology 9(2), 225-242 (2002)

2. Li, G., Liu, B., Xu, B.: Accurate Recognition of CIS-regulatory motifs with the Correct Lengths in Prokaryotic Genomes. Nucleic Acids Research 38(2), e12 (2009), http://nar.oxfordjournals.org

3. Fisher, P.S., Fisher, H., Baek, J., Angaye, C.: Syntactic Pattern Recognition Using Finite Inductive Strings. In: Kadirkamanathan, V., Sanguinetti, G., Girolami, M., Niranjan, M., Noirel, J. (eds.) PRIB 2009. LNCS (LNBI), vol. 5780, pp. 89-101. Springer, Heidelberg (2009)

4. Case, J.H., Fisher, P.S.: Long Term Memory Modules. Bulletin of Mathematical Biology 46(2), 295-326 (1984)

5. Source used for data in this paper, http://www.ncbi.nlm.nih.gov/nuccore/12751174

6. Smith, T.F., Waterman, M.S.: Identification of Common Molecular Subsequences. Journal of Molecular Biology 147, 195-197 (1981)

7. Fisher, P.S., Baek, J., Adeyeye, J., Setubal, J.: Finite Inductive Sequences, Kolmogorov Complexity with Application to Genome Sequences. In: International Conference on Bioinformatics, Computational Biology, Genomics and Chemoinformatics, BCBGC-10 (July 2010) 